\title{
Attenuation of Colitis by Serum-Derived Bovine Immunoglobulin/ Protein Isolate in a Defined Microbiota Mouse Model
}

\author{
Abigail L. Henderson ${ }^{1} \cdot$ Meghan Wymore Brand $^{2} \cdot$ Ross J. Darling ${ }^{2} \cdot$ \\ Kenneth J. Maas ${ }^{1,4} \cdot$ Christopher J. Detzel $^{1} \cdot$ Jesse Hostetter $^{3}$ • \\ Michael J. Wannemuehler ${ }^{2} \cdot$ Eric M. Weaver $^{1}$
}

Received: 17 April 2015/Accepted: 21 May 2015/Published online: 31 May 2015

(C) The Author(s) 2015. This article is published with open access at Springerlink.com

\begin{abstract}
Background The pathogenesis of inflammatory bowel disease (IBD) is complex and multifaceted including genetic predisposition, environmental components, microbial dysbiosis, and inappropriate immune activation to microbial components. Pathogenic bacterial provocateurs like adherent and invasive E. coli have been reported to increase susceptibility to Crohn's disease. Serum-derived bovine immunoglobulin/protein isolate (SBI) is comprised primarily of immunoglobulins (Igs) that bind to conserved microbial components and neutralize exotoxins.

Aim To demonstrate that oral administration of SBI may modulate mucosal inflammation following colonization with E. coli, LF82, and exposure to dextran sodium sulfate (DSS).

Methods Defined microbiota mice harboring the altered Schaedler flora (ASF) were administered SBI or hydrolyzed collagen twice daily starting 7 days prior to
\end{abstract}

Abigail L. Henderson and Meghan Wymore Brand are the first authors.

Electronic supplementary material The online version of this article (doi:10.1007/s10620-015-3726-5) contains supplementary material, which is available to authorized users.

Abigail L. Henderson

Abigail.henderson@enterahealth.com

1 Entera Health, Inc., 2575 SE Oak Tree Ct., Ankeny, IA 50023, USA

2 Department of Veterinary Microbiology and Preventive Medicine, College of Veterinary Medicine, Iowa State University, Ames, IA 50011, USA

3 Department of Veterinary Pathology, College of Veterinary Medicine, Iowa State University, Ames, IA 50011, USA

4 Entera Health, Inc., Cary, NC 27518, USA challenge with E. coli LF82 and continuing for the remainder of the experiment. Mice were treated with DSS for 7 days and then evaluated for evidence of local and peripheral inflammation.

Results Igs within SBI bound multiple antigens from all eight members of the ASF and E. coli LF82 by western blot analysis. Multiple parameters of LF82/DSS-induced colitis were reduced following administration of SBI, including histological lesion scores, secretion of cytokines and chemokines from cecal biopsies, intestinal fatty acid binding protein (I-FABP) and serum amyloid A from plasma.

Conclusions Oral administration of SBI attenuated clinical signs of LF82/DSS-induced colitis in mice. The data are consistent with the hypothesis that SBI immunoglobulin binding of bacterial antigens in the intestinal lumen may inhibit the inflammatory cascades that contribute to IBD, thus attenuating DSS-induced colitis.

Keywords Inflammatory bowel disease (IBD) $\cdot$ Dextran sulfate sodium (DSS) $\cdot$ Microbiota $\cdot$ Bovine immunoglobulin $\cdot$ Serum

\section{Introduction}

Inflammatory bowel diseases (IBD), including Crohn's disease (CD) and ulcerative colitis (UC), are a group of chronic intestinal disorders with a multifactorial etiology that involves complex interactions between the (GI) microbiota, genetics, environment, and the immune system [1]. The human GI tract is home to a diverse population of bacteria that reaches $10^{12}$ cells/gram contents in the colon [2-4]. Alterations in the gut microbiota (i.e., dysbiosis) resulting in a loss in microbial diversity are believed to 
contribute to an exaggerated adaptive immune response, further complicating the disease [5]. In addition, bacterial components or microbial-associated molecular patterns (MAMPS) have been shown to exacerbate the inflammation associated with IBD [6]. Mice with genetic susceptibilities to IBD (e.g., IL-10 ${ }^{-/-}$) do not develop IBD in a germfree environment [7]. However, germfree SAMP1/Yit mice develop spontaneous colitis and severe inflammation when housed in conventional environments [8].

Bacterial pathogens have been postulated to contribute to the severity of IBD. Notably adherent-invasive E. coli (AIEC) E. coli has been reported to enhance the pathogenesis and chronicity of adult and pediatric CD [9-14]. A well-characterized AIEC isolate, LF82, has been reported to increase colitis severity in mice administered DSS [15]. Several mechanisms might explain how AIEC contributes to the pathogenesis of $\mathrm{CD}$, including the presence of type 1 pili that facilitate attachment to mucosa and replication within host epithelial cells and macrophages without causing cell death $[16,17]$. These mechanisms may contribute to the downstream dysregulated immune reactivity toward the resident GI microbiota.

Multiple studies have shown that oral administration of animal plasma can reduce GI enteropathy and restore homeostasis in animals $[18,19]$. SBI is predominantly $\operatorname{IgG}$ $(>50 \%)$ and intended for oral consumption to provide distinctive nutritional requirements for the clinical dietary management of specific GI conditions under physician supervision (e.g., in irritable bowel syndrome with diarrhea (IBS-D), IBD, and HIV-associated enteropathy) [18]. We recently demonstrated that SBI binds to microbial components resulting in steric and immune exclusion mechanisms to modulate inflammation [20]. Specifically, the Ig component of SBI binds bacterial antigens to either (1) increase the size of the antigen to prevent translocation across an epithelial membrane (steric exclusion) or (2) block the recognition sites on antigens that are required to activate monocytes (immune exclusion) [20]. These in vitro findings supported the rationale that binding microbial antigens by Igs within SBI may help ameliorate the inflammation associated with IBD.

In this study, the impact of oral SBI administration on downstream intestinal inflammation was assessed in an LF82/DSS model of IBD. In this model, $\mathrm{C} 3 \mathrm{H} / \mathrm{HeN}$ mice harboring a defined intestinal microbiota were colonized with $E$. coli LF82 prior to low-dose DSS exposure to induce mucosal inflammation. Our results show that SBI administration reduced local and systemic inflammation associated with LF82/DSS-induced colitis. Where bacterial-mediated inflammation is operative in IBD patients, oral consumption of SBI and its microbial binding components may provide valuable benefits.

\section{Materials and Methods}

\section{Animals}

Male and female gnotobiotic $\mathrm{C} 3 \mathrm{H} / \mathrm{HeN}$ :TAC mice (12 weeks of age, 25-30 g) harboring the altered Schaedler flora (ASF) were bred and maintained under gnotobiotic conditions in flexible film isolators (housed at Iowa State University). Mice were exposed to a 12-h light/dark cycle, and an irradiated diet (Harlan 2919) and sterile water were provided ad libitum. Mice were monitored daily for dehydration, activity, ruffled fur, and diarrhea. The ASF consisted of the following bacterial members: ASF356, Clostridium sp.; ASF360, Lactobacillus intestinalis; ASF361, Lactobacillus murinus; ASF457, Mucispirillum schaedleri; ASF492, Eubacterium plexicaudatum; ASF500, Pseudoflavonifactor sp.; ASF502, Clostridium sp.; and ASF519, Parabacteroides goldsteinii [21].

\section{Experimental Design}

\section{Methods}

ASF $\mathrm{C} 3 \mathrm{H} / \mathrm{HeN}$ mice received $50 \mathrm{mg}$ of either SBI or $\mathrm{HC}$ as a control protein twice daily (AM and PM) by oral gavage $(100 \mathrm{mg} / \mathrm{mouse} /$ day), starting on day -7 for 5 weeks (Fig. 1). SBI was used to target bacterial antigens in the GI tract that would contribute to inflammation. Ten percent solutions $(10 \% \mathrm{w} / \mathrm{v})$ of SBI and $\mathrm{HC}(10 \%$ protein w/v) (Entera Health, Inc., Ankeny, IA) were prepared in PBS, centrifuged, and pre-filtered through a nominal filter $(0.5 \mu \mathrm{m}$, Millipore opticap XL 4 capsule \# KWSSA04HH3) followed by a $0.22-\mu \mathrm{m}$ filter (Nalgene

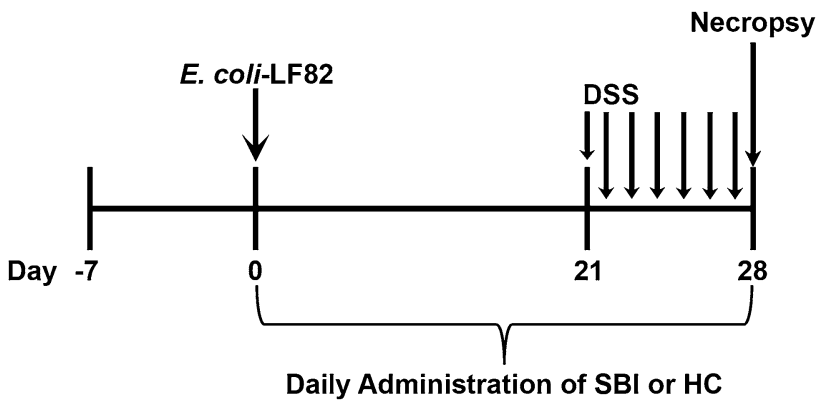

Fig. 1 Experimental design. Defined microbiota $\mathrm{C} 3 \mathrm{H} / \mathrm{HeN}$ mice were gavaged daily with either $100 \mathrm{mg}$ of serum-derived bovine immunoglobulin/protein isolate (SBI) or hydrolyzed collagen (HC). One week after protein administration was initiated mice were orally gavaged with $5 \times 10^{8} \mathrm{CFU}$ of $E$. coli LF82. Low-dose DSS ( $2 \%$ for the first 2 days and then $1.5 \%$ for the remaining 5 days) was administered in drinking water starting 3 weeks after $E$. coli colonization. Mice were allowed free access to the DSS for 7 days prior to necropsy 
\#569-0020). Sterility was confirmed on blood agar plates and trypticase soy broth under aerobic conditions. Solutions of SBI and HC were aseptically transferred into isolators for daily feedings. No adverse events were observed.

\section{Infection With E. coli LF82}

One week after administration of SBI and HC, mice were orally colonized with $5 \times 10^{8} \mathrm{CFU}$ of Escherichia coli LF82 obtained from Dr. Kenny Simpson (Cornell, Ithaca, NY) which was aerobically maintained on TSA plates at $37{ }^{\circ} \mathrm{C}$. On day zero, bacteria were harvested from plates and suspended in sterile PBS. Quantification of bacteria was estimated using the McFarland standards and confirmed with serial dilution of the inoculum. The suspension was aseptically transferred into the isolators, and mice were orally gavaged with $0.2 \mathrm{ml}$ of $E$. coli LF82 culture $\left(5 \times 10^{8}\right.$ CFUs). E. coli LF82 colonization was confirmed at necropsy by standard culture of cecal contents on TSA and MacConkey plates.

\section{DSS Treatment}

DSS (MP Biomedicals, LLC \# 160110) was prepared in sterile water and filtered $(0.2 \mu \mathrm{m})$ before aseptically transferred into isolators. Starting 3 weeks after E. coli colonization, mice received DSS in drinking water for 7 days ( $2 \%$ for the first 2 days and then $1.5 \%$ for the remaining 5 days). Mice were allowed to drink DSS water ad libitum. After euthanasia $\left(\mathrm{CO}_{2}\right.$ asphyxiation), tissues were evaluated macroscopically and processed for histopathology, cytokine, and inflammatory marker analysis.

\section{Preparation of Bacterial Antigens for Western Blots}

Bacteria (eight ASF members and LF82) were cultivated in BHI broth supplemented with bovine serum at $37^{\circ} \mathrm{C}$ in an anaerobic chamber. Bacteria were harvested, washed in PBS, frozen, lyophilized, and stored at $-20^{\circ} \mathrm{C}$. For western blot analysis, bacteria were resuspended in sterile PBS $(2 \mathrm{mg} / \mathrm{ml})$, frozen, and thawed at -20 and $-80{ }^{\circ} \mathrm{C}$ twice each, followed by sonication at maximum power on ice. The lysates were sterilized by ultraviolet irradiation and sterility confirmed bacteriologically.

\section{Macroscopic Scores}

Cecum and colon were harvested at necropsy and scored for gross inflammatory changes on a binary scale of $0-1$ for the presence/absence of the following criteria: cecal atrophy, enlarged cecal tonsil, cecal emptying, abnormally watery or mucoid intraluminal cecal, and/or colon contents, luminal blood in cecum, and/or colon, tissue thickening of cecum, and/or colon, and the absence of formed fecal pellets in colon. Individual scores were combined (maximum score of 10) to derive a macroscopic score.

\section{Histopathological Analysis}

Samples of cecum and colon were fixed in $10 \%$ neutral buffered formalin, paraffin embedded, sectioned, and stained with hematoxylin and eosin. Tissue sections were evaluated by a blinded, board-certified veterinary pathologist $(\mathrm{JH})$. Tissues were scored on severity of ulceration, inflammation, edema, stromal collapse, gland hyperplasia, and height of the mucosa. Each criterion was individually scored on a scale of $0-5$, except height of the mucosa which was measured as the average ratio of crypt height divided by crypt width (scale of 1-7). The total score is represented as the sum of all criteria (total score scale of $0-32$ ).

\section{Evaluation of Systemic Inflammatory and Damage Markers}

Plasma samples were obtained at necropsy and stored at $-20{ }^{\circ} \mathrm{C}$ until analysis. Serum amyloid A (SAA) analysis was performed using an ELISA kit (Life Technologies, \#KMA0021) at a 1:200 dilution. Intestinal fatty acid binding protein (I-FABP) in mouse plasma (diluted at 1:500) was assessed by ELISA (MyBioSource, \#MBS702875). Both SAA and I-FABP ELISAs were performed according to the manufacturer's instructions (microplate reader-Molecular Devices, Sunnyvale, CA).

\section{Evaluation of Mucosal Cytokine and Chemokine Levels}

One-centimeter cecal tissue biopsies were harvested at necropsy in triplicate from each animal, washed, and incubated in tissue culture medium (RMPI 1640) plus penicillin, streptomycin, gentamicin, glutamine, pyruvate, and 2-mercaptoethanol at $37{ }^{\circ} \mathrm{C}$. Supernatants were harvested from cecal biopsies at $24 \mathrm{~h}$, and triplicate samples from individual animals were pooled and frozen at $-20{ }^{\circ} \mathrm{C}$ until analysis. A multiplex bead-based assay from Bio-Rad (BioPlex Pro ${ }^{\mathrm{TM}}$, Bio-Rad, Hercules, CA) assessed levels of mouse cytokines/chemokines including IL-6, G-CSF, and CCL5 using a Bio-Plex ${ }^{\circledR}$ MAGPIX ${ }^{\mathrm{TM}}$ Multiplex Reader, Bio-Rad.

\section{Western Blot}

ASF and LF82 bacterial antigens were standardized for protein content by BCA protein assay (Pierce, Rockford, IL, \#23227). Antigen samples $(5 \mu \mathrm{g})$ were reduced by 
boiling in LDS buffer (Life Technologies, \#NP0008) and Bolt reducing agent (Life Technologies, \#B0009) and separated on $4-12 \%$ Bis/Tris gels (Life Technologies, \#NP0336PK2). Gels were silver stained to demonstrate equalized protein and loading. Following confirmation of equal protein by BCA and silver stain, additional gels were blotted onto a PVDF membrane and blocked with Pierce protein-free T20 blocking buffer. The membrane was then blotted with $0.1 \%$ SBI overnight and incubated with rabbit anti-bovine IgG HRP at 1:25,000 (Sigma, \#A5295). The blot was visualized with SuperSignal West Pico chemiluminescent substrate (Pierce, \#34087) using a C-DiGit (LICOR, Lincoln, NE).

\section{Statistical Analysis}

Actual mice used per treatment group were as follows: (1) $\mathrm{HC} / \mathrm{LF} 82=7, \quad$ (2) $\mathrm{HC} / \mathrm{LF} 82+\mathrm{DSS}=10, \quad$ (3) $\mathrm{SBI} /$ $\mathrm{LF} 82=8$, and (4) SBI/LF82 + DSS $=11$. All mice were analyzed for each assay. Mice were assigned to a treatment group based on sex and number of mice per cage. The order in which the mice were treated each day rotated between the various cages. A two-way analysis of variance (ANOVA) was performed on all four treatment groups (7-11 mice per group), and t-tests were utilized when significantly different groups were identified by ANOVA. Each mouse was an experimental unit.

\section{Ethical Considerations}

All mouse experiments were performed according to the requirements of the Iowa State University Institutional Animal Care and Use Committee (IACUC) with the following approval number: 9-04-5755-M.

\section{Results}

\section{Colonization of Defined Microbiota C3H Mice with $E$. coli LF82}

Prior to treatments, all mice were healthy and free of extraneous contaminants. Gnotobiotic $\mathrm{C} 3 \mathrm{H} / \mathrm{HeN}$ :TAC mice harboring the ASF became chronically colonized with E. coli LF82 after a single oral gavage. At necropsy, all mice were positive for E. coli LF82 by culture of cecal contents and CFUs were not altered by DSS administration (supplemental data, S1). Three environmental aerobic bacterial contaminants were found to have been unintentionally introduced into the gnotobiotic isolators, Stenotrophomonas, Pseudomonas, and Acinetobacter. While all mice were likely exposed to the contaminants, multiple cultures of fecal/cecal contents were negative for the growth of these organisms, indicating that the quantity of vegetative bacteria in the GI tract was too low to culture. The low abundance of each contaminant $(\leq 1 \%)$ was detected in the majority of mice through fecal DNA sequencing (data not shown).

\section{Binding of SBI to Altered Schaedler Flora and $E$. coli Lysates}

SBI predominately consists of $\operatorname{IgG}(>50 \%)$ and has been shown to bind and neutralize conserved bacterial antigens and toxins in vitro $[20,22,23]$. It was hypothesized that SBI would bind to bacterial antigens in the intestinal lumen and attenuate the severity of DSS-induced inflammation. To demonstrate that SBI was capable of binding to ASF antigens, individual ASF bacterial lysates were separated by SDS-PAGE, transferred to membranes, and reacted with SBI. As shown in Fig. 2, bovine (IgG) in SBI recognized multiple antigenic components from each of the ASF bacterial lysates and E. coli LF82.

\section{SBI Attenuated Macroscopic and Histological Changes}

LF82/DSS mice administered HC had elevated intestinal macroscopic scores compared with HC-gavaged mice colonized with LF82 alone (Fig. 3, $p=0.02$ ). The administration of SBI to the LF82/DSS mice trended toward a reduction in the macroscopic score compared with HCgavaged LF82/DSS mice (Fig. 3, $p=0.14$ ). In the absence of DSS exposure, the observed differences in the macroscopic lesions between mice treated with HC or SBI were minimal (Fig. 3). Colonic and cecal histological scores were increased in LF82/DSS mice given HC compared with LF82-colonized mice administered HC (Figs. 4a, b, $5 \mathrm{a}, \mathrm{b})$. The DSS-induced histological changes were characterized by increased mucosal height, ulceration, edema, glandular hyperplasia, as well as increases in inflammatory cell infiltrate consisting of mononuclear and polymorphonuclear leukocytes in the colon and cecum (Figs. 4b, 5b). Administration of SBI lessened the histological changes in the colon (Fig. 4d) and cecum (Fig. 5d) of LF82/DSS mice. The overall histopathological score of LF82/DSS-induced epithelial damage and inflammation in both the colon (Fig. 4e) and cecum (Fig. 5e) was significantly decreased in animals given SBI compared with those administered HC $(p<0.05)$.

\section{Evaluation of Cytokine and Chemokines From Cecal Biopsies}

The release of cytokines and chemokines from cecal explants was assessed to determine the potential impact of SBI binding 
Fig. 2 SBI binding of altered Schaedler flora (ASF) and LF82 bacterial antigens. ASF wholecell lysates were separated by SDS-PAGE and transferred to a nitrocellulose membrane. Lane 1 protein standard, 2 ASF 356, 3 ASF 360, 4 ASF 361, 5 ASF 457, 6 ASF 492, 7 ASF 500, 8 ASF 502, 9 ASF 519, and 10 LF82. Membrane was blotted with a $0.1 \%$ serum-derived bovine immunoglobulin/protein isolate (SBI) solution followed by a 1:25,000 dilution of antibovine IgG HRP and visualized with chemiluminescence. Left: western blot and right: silver stain

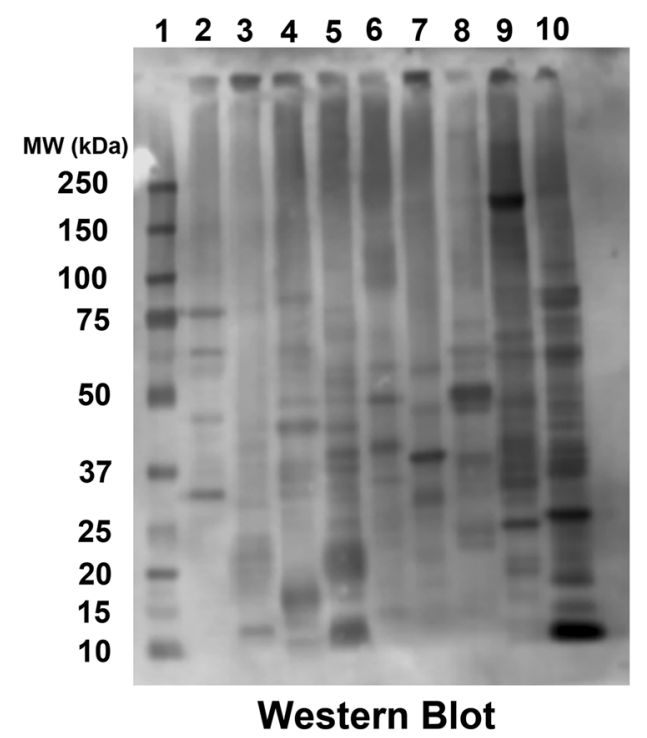

$\begin{array}{llllllllll}1 & 2 & 3 & 4 & 5 & 6 & 7 & 8 & 9 & 10\end{array}$

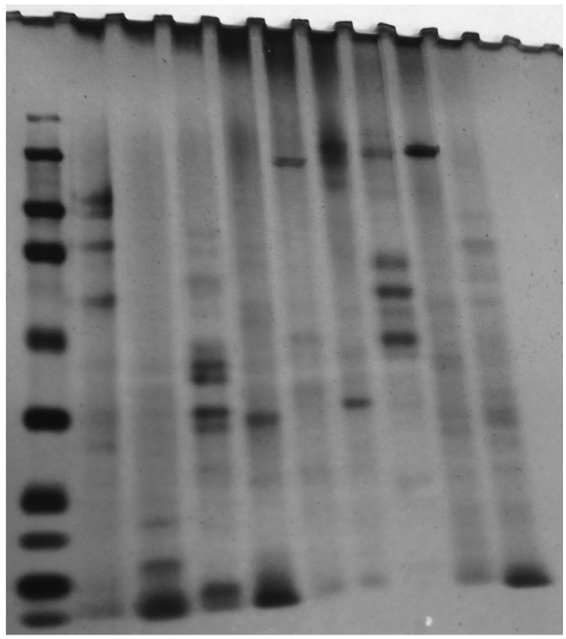

Silver Stain

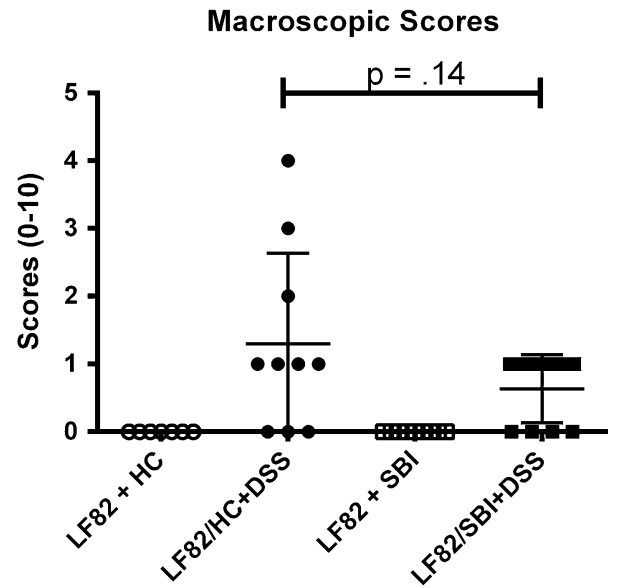

Fig. 3 Intestinal macroscopic score. Scores were evaluated at necropsy from ASF-harboring mice challenged with E. coli (LF82) with or without DSS and gavaged with either serum-derived bovine immunoglobulin (SBI) or hydrolyzed collagen (HC). Scores were determined by the presence or absence of gross anatomic changes indicative of inflammatory responses in cecum and colon as described in "Materials and Methods" section. Two-way ANOVA indicated that macroscopic score was affected by DSS administration ( $p=0.0005)$, but not affected by protein choice $(p=0.1947)$. The interaction of the two variables on macroscopic score was not significant ( $p=0.1947)$ following two-way ANOVA

to bacterial components on mucosal inflammation. The administration of SBI to LF82/DSS mice resulted in significant reductions in IL-6 secretion from cecal biopsies compared with HC-gavaged mice LF82/DSS (Fig. 6, $p=0.006$ ). Based on the increased cellular infiltrate observed in histological sections of the LF82/DSS mice, the secretion of CCL5 and G-CSF was also measured. CCL5 is a chemokine that recruits monocytes, lymphocytes, mast cells, and eosinophils and has been shown to be upregulated by cells within the subepithelial lamina propria following activation by microbial antigens, as well as by DSS- and TNBS-induced colitis [24, 25]. LF82/ DSS administration increased the secretion of CCL5 from cecal explants in the HC-treated group (Fig. 7, mean = $108 \mathrm{pg} / \mathrm{ml}$ ) compared with mice given HC alone (Fig. 7, mean $=49$ ). Although not statistically significant, there was a strong trend toward decreased levels of CCL5 in LF82/DSS mice administered SBI (Fig. 7, mean $=49 \mathrm{pg} / \mathrm{ml}$ ) that were similar to levels observed in LF82-colonized mice given HC (Fig. 7, mean $=49 \mathrm{pg} / \mathrm{ml}$ ). A similar trend was observed for G-CSF, a chemokine that functions to recruit and differentiate granulocytes into neutrophils (Fig. 7, mean of HC and LF82/ $\mathrm{DSS}=6569 \mathrm{pg} / \mathrm{ml} ;$ mean of SBI and LF82/DSS $=$ $5163 \mathrm{pg} / \mathrm{ml})$.

\section{Attenuation of Intestinal Injury with SBI}

In clinical and experimental colitis, epithelial cell functions are altered as a consequence of damage to the gut [26]. I-FABP is a cytoplasmic protein found in epithelial cells of the small intestine and proximal colon and has proven an excellent blood biomarker of intestinal injury and ischemia in IBD [27-29]. Compared with mice treated with HC, plasma I-FABP levels were found to be significantly decreased following SBI administration (Fig. 8, $p=0.03$ ) in mice colonized with LF82 but not treated with DSS. For LF82/DSS mice, administration of SBI resulted in significantly lowered levels of I-FABP compared with LF82/ DSS mice given HC (Fig. 8, $p=0.0003$ ).

\section{Attenuation of a Systemic Marker of Inflammation in the Presence of SBI}

SAA is an acute-phase protein secreted primarily by the liver during inflammation. To determine whether peripheral 


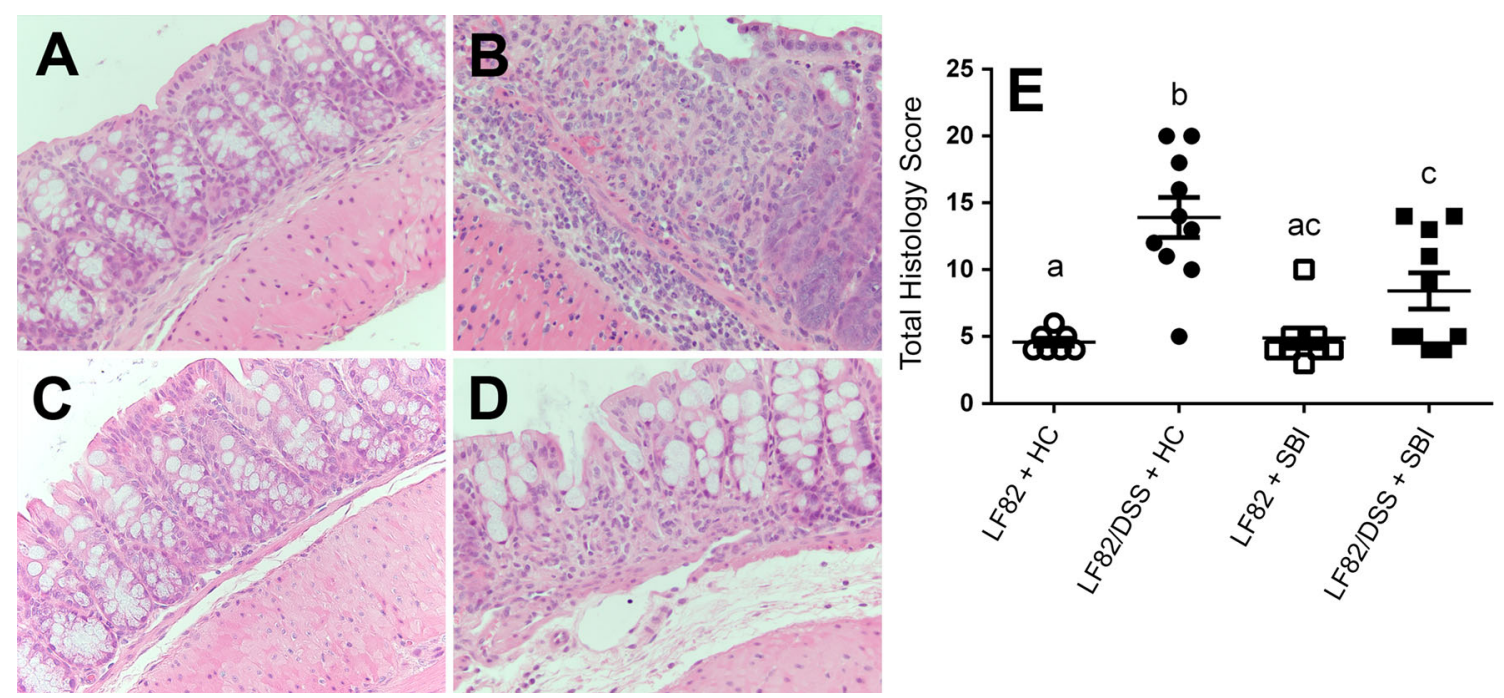

Fig. 4 Histopathological scores of colon tissue. Colons were recovered from E. coli LF82-colonized defined microbiota mice treated with a hydrolyzed collagen (HC), b HC + DSS showing increased mucosal height, gland hyperplasia, ulcerated bed, swollen endothelium, and some blood, c serum-derived bovine immunoglobulin/ protein isolate (SBI), or d SBI + DSS depicting less inflammatory infiltrate, gland hyperplasia, and stromal collapse than HC + DSS. e Comparison of histopathological scores among treatment groups; lesions were scored in a blinded fashion with a maximum histological score (summation of individual parameters) of 32. Groups with identical letters are not statistically different from each other $(p \leq 0.05)$. All images are $\times 400$. Two-way ANOVA indicated that colon scores were affected by both the administration of DSS $(p=0.0001)$ and protein choice $(p=0.0435)$. There was also a significant interaction found between the two variables on colon score following two-way ANOVA ( $p=0.0253$ )
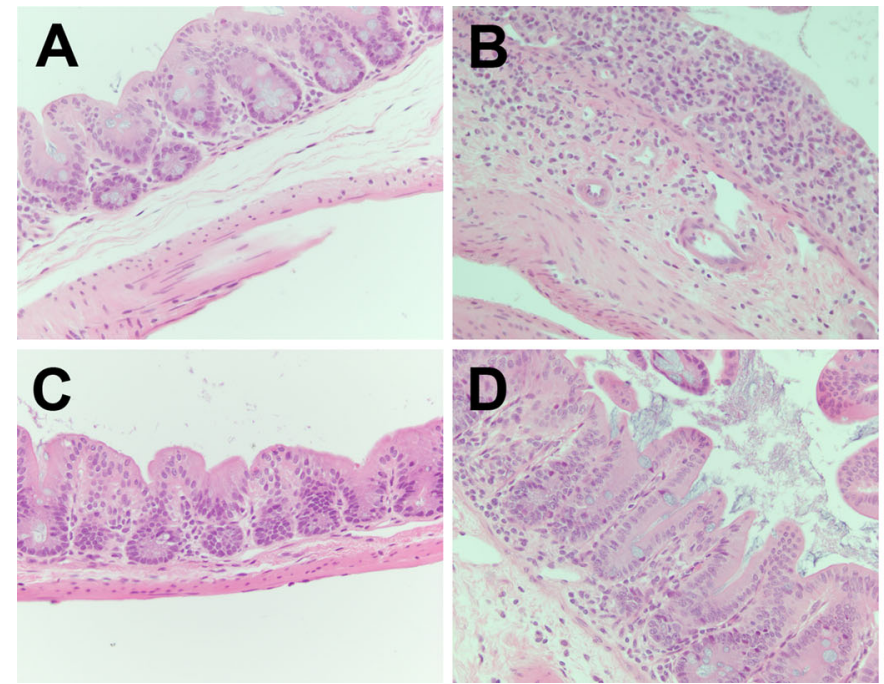

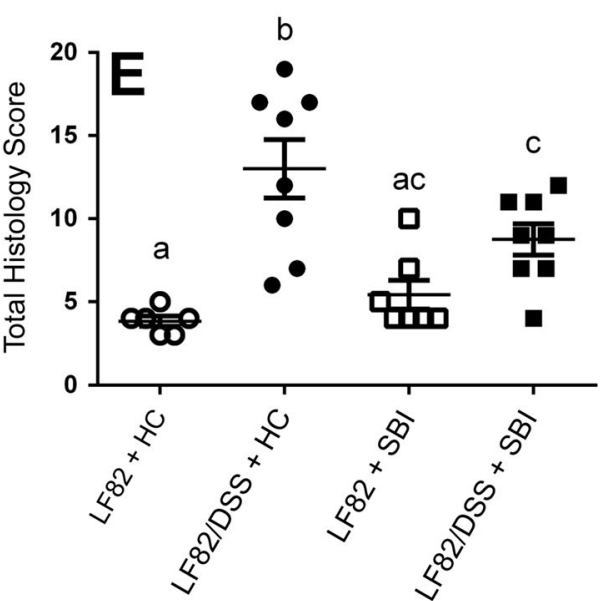

scored in a blinded fashion with a maximum histological score (summation of individual parameters) of 32. Groups with identical letters are not statistically different from each other $(p \leq 0.05)$. All images are $\times 400$. Two-way ANOVA indicated that cecal scores were affected by the administration of DSS $(p=0.0001)$, but not protein choice $(p=0.2780)$. There was a significant interaction found between the two variables on cecal score following two-way ANOVA $(p=0.0220)$

mice colonized with LF82 alone (Fig. 9, $p=0.002$ ). However, SAA levels in LF82/DSS mice administered SBI remained at control levels and were significantly lower than LF82/DSS mice gavaged with HC (Fig. 9, $p=0.0006$ ). 


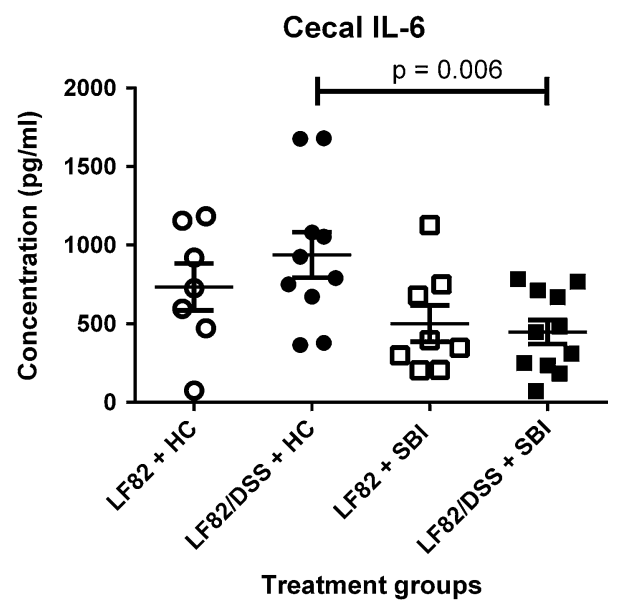

Fig. 6 Concentration of IL-6 from cecal biopsies. Culture supernatants were collected after $24 \mathrm{~h}$ of incubation of cecal biopsies in media collected from E. coli LF82-colonized defined microbiota mice were treated with hydrolyzed collagen (HC), HC + DSS, serumderived bovine immunoglobulin/protein isolate (SBI), or SBI + DSS. Two-way ANOVA indicated that IL-6 concentration was affected by protein choice $(p=0.0061)$, but not DSS administration $(p=0.5442)$. The interaction of the two variables on IL-6 concentration was not significant $(p=0.3014)$ following two-way ANOVA. Further analysis indicated that SBI significantly reduced IL-6 concentration in the LF82/DSS colitis model (HC + LF82/DSS compared with SBI + LF82/DSS, $p=0.006$ )

\section{Discussion}

As reported previously, SBI has been shown to bind and neutralize bacterial antigens in vitro resulting in a reduction in inflammatory cytokines produced from monocytes in a transwell system [20]. The present study was undertaken to expand upon those initial findings and to assess the effect of SBI on inflammation in a mouse model of colitis where a bacterial provocateur was administered. In experiments reported here, results indicate a reduction in epithelial damage as well as decreased mucosal and systemic inflammation in LF82/DSS-treated animals when administered SBI versus a control protein, HC.

The DSS-induced colitis model in mice was utilized because it is an analog model for studying human IBD, and most closely resembles UC [30]. Similar to human UC, DSS treatment induces epithelial damage, inflammatory cell infiltrate, and symptoms of watery and/or bloody diarrhea [30]. Using a "multiple-hit" colitis model that includes colonization of defined microbiota mice with a novel organism (e.g., Helicobacter bilis), previous work has demonstrated increases in host susceptibility to lowdose DSS-induced colitis resulting in more severe inflammatory changes [32]. E. coli LF82 is an AIEC isolate from a patient with ileal CD $[9,10]$. As previously reported, DSS treatment increased the severity of colitis in mice colonized with $E$. coli LF82, which may be due to the ability of E. coli LF82 to disrupt the integrity of the epithelial barrier $[15,33]$. In mice harboring a conventional GI microbiota, E. coli LF82 has not been shown to successfully colonize without repeated administration [15, 34]. In contrast, defined microbiota mice harboring the ASF become chronically colonized with $E$. coli LF82 following a single oral gavage. Accordingly, E. coli LF82 was used as the bacterial provocateur in the studies reported here to

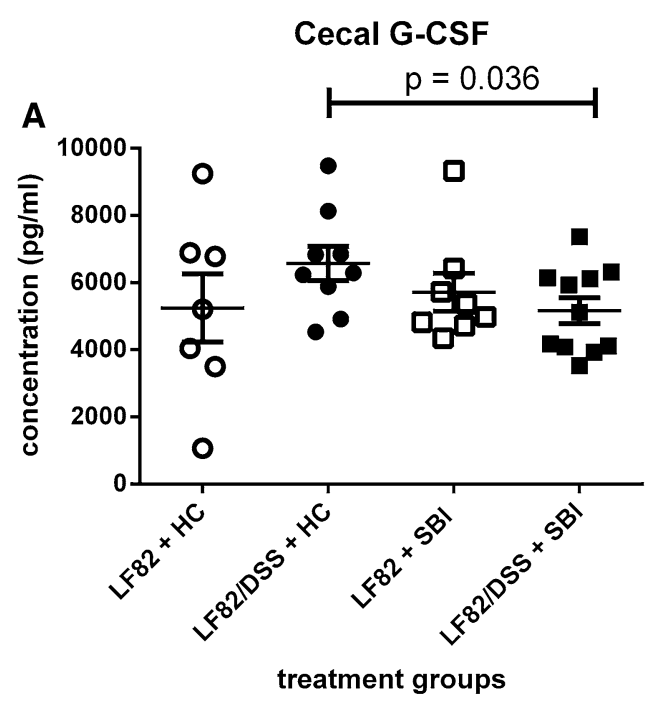

Fig. 7 Secretion of G-CSF (a) and CCL5 (b) from cecal biopsies. Culture supernatants were collected after $24 \mathrm{~h}$ of incubation of cecal biopsies in medium. Tissues were collected from E. coli LF82colonized defined microbiota mice treated with hydrolyzed collagen (HC), HC + DSS, serum-derived bovine immunoglobulin/protein

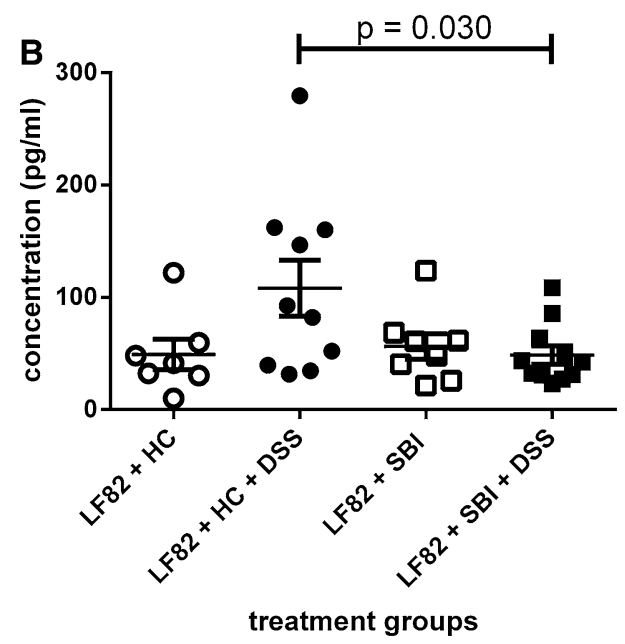

isolate (SBI), or SBI + DSS. For panels A and B, Two-way ANOVA indicated that the addition of DSS or protein did not significantly alter secretion of G-CSF or CCL5. Further analysis indicated a strong trend, but not significant interaction of the two variables on G-CSF $(p=0.1302)$ or CCL5 concentration $(p=0.0558)$ 
Intestinal Fatty Acid Binding Protein

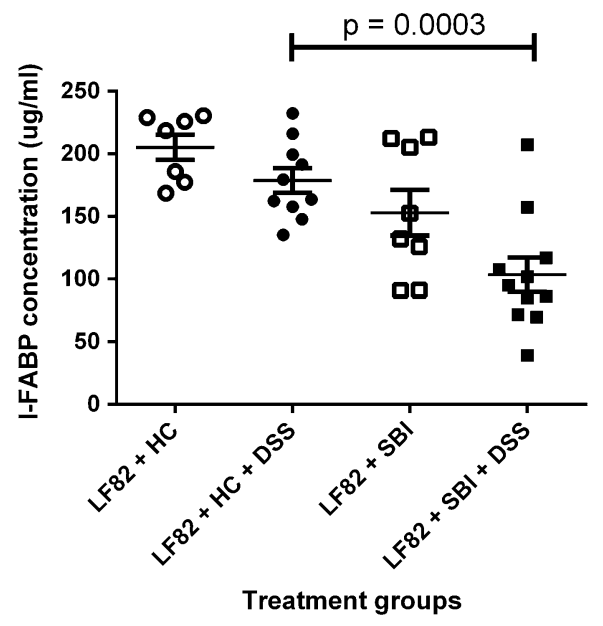

Fig. 8 Evaluation of intestinal fatty-acid-binding protein (I-FABP). I-FABP from plasma was analyzed as an indicator of intestinal damage. E. coli LF82-colonized defined microbiota mice were treated with hydrolyzed collagen (HC), HC + DSS, serum-derived bovine immunoglobulin/protein isolate (SBI), or SBI + DSS. Plasma was collected at necropsy and assayed for I-FABP by ELISA. Two-way ANOVA indicated that I-FABP concentration was affected by both the administration of DSS $(p=0.0093)$ and protein choice $(p=0.0001)$. However, the interaction of the two variables on I-FABP concentration was not significant $(p=0.4119)$. Further analysis indicated that SBI significantly reduced I-FABP concentration in the LF82/DSS colitis model (HC + LF82/DSS compared with SBI + LF82/DSS, $p=0.0003$ )

\section{Serum Amyloid A}

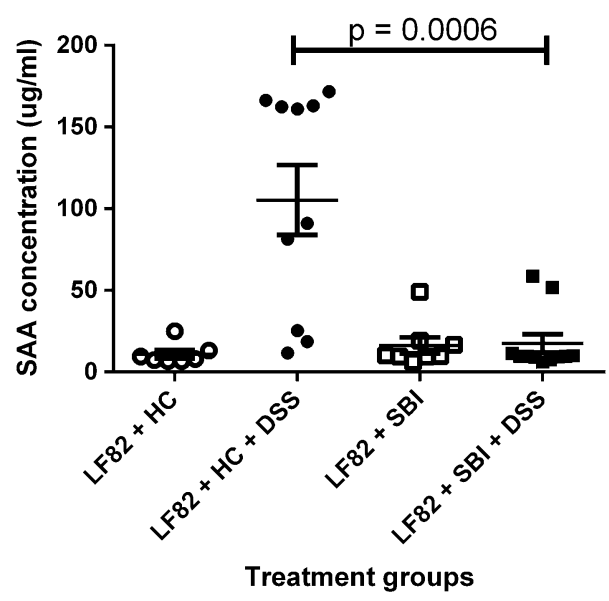

Fig. 9 Plasma serum amyloid A (SAA) levels as an indicator of inflammation. E. coli LF82-colonized defined microbiota mice were treated with hydrolyzed collagen (HC), HC + DSS, serum-derived bovine immunoglobulin/protein isolate (SBI), or SBI + DSS. Plasma was collected at necropsy and assayed for SAA by ELISA. Two-way ANOVA indicated that SAA concentration was affected by both the administration of DSS $(p=0.0008)$ and protein choice $(p=0.0031)$. Also, the interaction of the two variables on SAA concentration was significant $(p=0.0010)$. Further analysis indicated that SBI significantly reduced SAA concentration in the LF82/DSS colitis model $(\mathrm{HC}+\mathrm{LF} 82 / \mathrm{DSS}$ compared with SBI $+\mathrm{LF} 82 / \mathrm{DSS}, p=0.0006)$ heighten the sensitivity of the murine host to low-dose DSS.

Altered intestinal permeability increases both the susceptibility to disease and predicts relapse in $\operatorname{CD}[35,36]$. During both IBD and DSS-induced colitis, the epithelial barrier is compromised leading to increased epithelial permeability and translocation of bacterial products $[30$, 37]. In this study, SBI attenuated epithelial damage in the LF82/DSS multiple-hit model of colitis, as evidenced by reduced histopathological lesion scores and reduced I-FABP compared with mice given HC and LF82/DSS. I-FABP has been proposed as a plasma biomarker for intestinal injury in UC patients since levels were elevated in patients with severe disease [29]. However, in murine models, DSS-induced colitis is accompanied by decreased water and electrolyte absorption as well as transport of fatty acids. DSS decreased I-FABP in the colon during active disease and colonic enterocytes regain I-FABP function during the regenerative phase [38]. These results indicate that in the presence of SBI there is an attenuation of epithelial injury in LF82/DSS-induced colitis.

Mucosal inflammation typically occurs after a compromise in epithelial barrier and is another hallmark of chronic and relapsing IBD. This study demonstrated attenuation of DSS-induced inflammation in ASF mice colonized with E. coli LF82 when SBI was administered compared to HC. Evidence in reduced LF82/DSS-induced local inflammation was evident in histological sections of the GI tract along with mucosal IL- 6 production from cecal biopsies. IL-6 is a pleiotropic cytokine that is secreted from many cell types including macrophages, epithelial cells and $\mathrm{T}$ cells following inflammatory insult and functions include initiating the acute-phase response, activating SAA, and activating lymphocytes to differentiate into Th17 cells [39, 40]. In IBD patients, there is a dysregulation of the IL-6 pathway that correlates with disease progression [39]. IL-6 along with TGF $\beta$ help to differentiate effector Th17 cells and if dysregulated, lead to an imbalance in effector/ regulatory $\mathrm{T}$ cell functions implicated in the pathogenesis of chronic inflammation [41]. In this study, mucosal IL-6 production was reduced in the SBI administered LF82/DSS mice compared with the HC, LF82/DSS group. Future studies will address whether the decreased IL- 6 production was accompanied by a reduction in Th17 cells.

In addition, there was a reduction in several markers of acute inflammation following SBI administration. SAA is an acute-phase protein secreted by the liver and extrahepatic tissues, including the intestinal epithelium, and is considered a biomarker for inflammation [42]. SAA has been reported to be elevated in intestinal biopsies from $\mathrm{CD}$ patients and has also been shown to be induced by IL-6 [43-45]. In murine models of IBD, SAA levels precede clinical signs of inflammation as well as correlate with 
increasing severity of DSS-induced inflammation [46, 47]. In this study, there was a reduction in inflammation as evidenced by decreased levels of SAA when SBI was administered to LF82/DSS mice compared with LF82/DSS mice given $\mathrm{HC}$.

Previous studies have shown that the immunoglobulin component of SBI binds to bacterial antigens, including MAMPS as well as bacterial toxins [22, 23]. In a transwell co-culture model, SBI was shown to bind LPS, lipid A or Pam3CSK4 on the apical surface to prevent subsequent activation by THP-1 monocytes in the basal layer following damage to the membrane [20]. Western blot analysis presented herein demonstrates that SBI binds to multiple antigens associated with all eight members of the ASF as well as E. coli LF82. A hypothesis for the downstream effect of SBI binding to luminal antigens in vivo is to prevent these components from stimulating immune responses. Numerous reports exist in the literature explaining how alterations of the microbiota correlate with inflammation. The DSS model, along with other models of induced or spontaneous colitis (e.g., IL-10 ${ }^{-1-}$ mice), have reported that the resident microbiota has a central role in modulating the severity of clinical signs [7, 48-55]. Animals reared in conventional compared to germfree environments have increased evidence of inflammation. Furthermore, mice genetically susceptible to IBD have a reduction in severity of disease when treated with broad spectrum antibiotics. The endpoints measured in the SBI administered LF82/DSS mice indicate that SBI attenuated epithelial damage and inflammation in comparison to the HC administered LF82/DSS mice and are similar to what would be expected based on studies reducing the damaging effects of microbial components in a leaky gut. Thus, the reduction in inflammation seen in SBI administered LF82/ DSS mice may be explained by the extrapolation of the in vitro effects of SBI binding and preventing microbial components from activating immune responses which cause epithelial damage and inflammation.

Other experiments which utilized the DSS-induced colitis model also demonstrated improved disease activity scores and epithelial healing with oral bovine colostrum or serum Igs [56]. Further analysis of the spleen in these studies indicated that myeloid-derived suppressor cells $\left(\mathrm{CD} 11 \mathrm{~b}^{+-}\right.$ $\mathrm{Gr}^{+}$) that enhance recovery after colitis, were significantly increased in sIgA-fed mice [56]. These results indicate that the Ig component of bovine colostrum may have a positive effect on the management of colitis. Bovine colostrum and SBI are similar in that both contain growth factors and Igs; however, SBI is more highly enriched for Igs [57, 58]. We hypothesize that the Ig components within SBI may modulate inflammation by binding and preventing luminal antigens from translocating across a permeabilized epithelium, thereby reducing the recruitment of inflammatory effector cells and indirectly helping to promote mucosal healing and nutrient absorption. Future studies will assess the impact of SBI on influencing regulatory cell recruitment following LF82/DSS-induced inflammation.

In conclusion, a beneficial effect of SBI administration was demonstrated in a mouse model of E. coli LF82 and DSS-induced colitis. The Igs in SBI bound to antigens of the enteric microbiota and daily administration of SBI resulted in less damage and inflammation after challenging with LF82/DSS. A control protein, HC, did not have the same effect in this model suggesting the distinctive nature of the protein nutrients in SBI are uniquely required to manage the intestinal condition in this animal model of colitis. The ability of SBI to maintain mucosal health makes it an attractive agent to attenuate bacterial antigeninduced GI inflammation associated with human IBD.

Acknowledgments We thank Dr. Greg Phillips and Allie Proctor for their assistance in sequencing fecal samples.

Conflict of interest This work was funded by Entera Health Inc. Entera Health employees (AH, KM,CD,and EW) were additionally provided salary support. Study design, data collection and analysis, decision to publish and manuscript preparation was at the discretion of the authors (AH, MWB, RD, KM, CD,JH, MW and EW) and was not impacted by the funder.

Open Access This article is distributed under the terms of the Creative Commons Attribution-NonCommercial 4.0 International License (http://creativecommons.org/licenses/by-nc/4.0/), which permits any noncommercial use, distribution, and reproduction in any medium, provided you give appropriate credit to the original author(s) and the source, provide a link to the Creative Commons license, and indicate if changes were made.

\section{References}

1. Sartor RB. Mechanisms of disease: pathogenesis of Crohn's disease and ulcerative colitis. Nat Clin Pract Gastroenterol Hepatol. 2006;3:390-407.

2. Eckburg PB, Bik EM, Bernstein CN, et al. Diversity of the human intestinal microbial flora. Science. 2005;308:1635-1638.

3. Whitman WB, Coleman DC, Wiebe WJ. Prokaryotes: the unseen majority. Proc Natl Acad Sci USA. 1998;95:6578-6583.

4. Guarner F, Malagelada JR. Gut flora in health and disease. Lancet. 2003;361:512-519.

5. Manichanh C, Rigottier-Gois L, Bonnaud E, et al. Reduced diversity of faecal microbiota in Crohn's disease revealed by a metagenomic approach. Gut. 2006;55:205-211.

6. Caradonna L, Amati L, Magrone T, Pellegrino NM, Jirillo E, Caccavo D. Enteric bacteria, lipopolysaccharides and related cytokines in inflammatory bowel disease: biological and clinical significance. J Endotoxin Res. 2000;6:205-214.

7. Sellon RK, Tonkonogy S, Schultz M, et al. Resident enteric bacteria are necessary for development of spontaneous colitis and immune system activation in interleukin-10-deficient mice. Infect Immun. 1998;66:5224-5231. 
8. Matsumoto S, Okabe Y, Setoyama H, et al. Inflammatory bowel disease-like enteritis and caecitis in a senescence accelerated mouse P1/Yit strain. Gut. 1998;43:71-78.

9. Darfeuille-Michaud A, Boudeau J, Bulois P, et al. High prevalence of adherent-invasive Escherichia coli associated with ileal mucosa in Crohn's disease. Gastroenterology. 2004;127: 412-421.

10. Darfeuille-Michaud A, Neut C, Barnich N, et al. Presence of adherent Escherichia coli strains in ileal mucosa of patients with Crohn's disease. Gastroenterology. 1998;115:1405-1413.

11. Sasaki M, Sitaraman SV, Babbin BA, et al. Invasive Escherichia coli are a feature of Crohn's disease. Lab Invest. 2007;87: 1042-1054.

12. Rolhion N, Darfeuille-Michaud A. Adherent-invasive Escherichia coli in inflammatory bowel disease. Inflamm Bowel Dis. 2007; 13:1277-1283.

13. Martinez-Medina M, Aldeguer X, Lopez-Siles M, et al. Molecular diversity of Escherichia coli in the human gut: new ecological evidence supporting the role of adherent-invasive $E$. coli (AIEC) in Crohn's disease. Inflamm Bowel Dis. 2009;15: 872-882.

14. Conte MP, Longhi $\mathrm{C}$, Marazzato $\mathrm{M}$, et al. Adherent-invasive Escherichia coli (AIEC) in pediatric Crohn's disease patients: phenotypic and genetic pathogenic features. BMC Res Notes. 2014;7:748

15. Carvalho FA, Barnich N, Sauvanet P, Darcha C, Gelot A, Darfeuille-Michaud A. Crohn's disease-associated Escherichia coli LF82 aggravates colitis in injured mouse colon via signaling by flagellin. Inflamm Bowel Dis. 2008;14:1051-1060.

16. Chassaing B, Darfeuille-Michaud A. The commensal microbiota and enteropathogens in the pathogenesis of inflammatory bowel diseases. Gastroenterology. 2011;140:1720-1728.

17. Glasser AL, Boudeau J, Barnich N, Perruchot MH, Colombel JF, Darfeuille-Michaud A. Adherent invasive Escherichia coli strains from patients with Crohn's disease survive and replicate within macrophages without inducing host cell death. Infect Immun. 2001;69:5529-5537.

18. Petschow BW, Blikslager AT, Weaver EM, et al. Bovine immunoglobulin protein isolates for the nutritional management of enteropathy. World J Gastroenterol. 2014;20:11713-11726.

19. Petschow BW, Burnett B, Shaw AL, Weaver EM, Klein GL. Serum-derived bovine immunoglobulin/protein isolate: postulated mechanism of action for management of enteropathy. Clin Exp Gastroenterol. 2014;7:181-190.

20. Detzel CJ, Horgan A, Henderson AL, Petschow BW, Warner CD, Maas KJ, Weaver EM. Bovine immunoglobulin/protein isolate binds pro-inflammatory bacterial compounds and prevents immune activation in an intestinal co-culture model. PLoS One. 2015;10(4) doi: 10.1371/journal.pone.0120278.

21. Wannemuehler MJ, Overstreet AM, Ward DV, Phillips GJ. Draft genome sequences of the altered Schaedler flora, a defined bacterial community from gnotobiotic mice. Genome Announce. $2014 ; 2$.

22. Horgan A, Maas K, Henderson A, Detzel C, Weaver E. Serumderived bovine immunoglobulin/protein isolate binds to microbeassociated molecular patterns. Experimental biology. Washington, DC: Federation of American Societies For Experimental Biology; 2014.

23. Henderson A, Horgan A, Detzel C, Maas K, Weaver E. Serumderived bovine immunoglobulin/protein isolate binds and neutralizes Clostridium difficile toxins A and B. Digestive Disease Week. 2014.

24. Elinav E, Strowig T, Kau AL, et al. NLRP6 inflammasome regulates colonic microbial ecology and risk for colitis. Cell. 2011;145:745-757.
25. Ajuebor MN, Hogaboam CM, Kunkel SL, Proudfoot AE, Wallace JL. The chemokine RANTES is a crucial mediator of the progression from acute to chronic colitis in the rat. $J$ Immunol. 2001;166:552-558.

26. Krishnan K, Arnone B, Buchman A. Intestinal growth factors: potential use in the treatment of inflammatory bowel disease and their role in mucosal healing. Inflamm Bowel Dis. 2011;17: 410-422.

27. Pelsers MM, Hermens WT, Glatz JF. Fatty acid-binding proteins as plasma markers of tissue injury. Clin Chim Acta. 2005;352:15-35.

28. Shores DR, Binion DG, Freeman BA, Baker PR. New insights into the role of fatty acids in the pathogenesis and resolution of inflammatory bowel disease. Inflamm Bowel Dis. 2011;17: 2192-2204.

29. Wiercinska-Drapalo A, Jaroszewicz J, Siwak E, Pogorzelska J, Prokopowicz D. Intestinal fatty acid binding protein (I-FABP) as a possible biomarker of ileitis in patients with ulcerative colitis. Regul Pept. 2008;147:25-28.

30. Okayasu I, Hatakeyama S, Yamada M, Ohkusa T, Inagaki Y, Nakaya R. A novel method in the induction of reliable experimental acute and chronic ulcerative colitis in mice. Gastroenterology. 1990;98:694-702.

31. Boismenu R, Chen Y. Insights from mouse models of colitis. $J$ Leukoc Biol. 2000;67:267-278.

32. Liu Z, Ramer-Tait AE, Henderson AL, et al. Helicobacter bilis colonization enhances susceptibility to typhlocolitis following an inflammatory trigger. Dig Dis Sci. 2011;56:2838-2848.

33. Wine E, Ossa JC, Gray-Owen SD, Sherman PM. Adherent-invasive Escherichia coli, strain LF82 disrupts apical junctional complexes in polarized epithelia. BMC Microbiol. 2009;9:180.

34. Carvalho FA, Barnich N, Sivignon A, et al. Crohn's disease adherent-invasive Escherichia coli colonize and induce strong gut inflammation in transgenic mice expressing human CEACAM. $J$ Exp Med. 2009;206:2179-2189.

35. D'Inca R, Annese V, di Leo V, et al. Increased intestinal permeability and NOD2 variants in familial and sporadic Crohn's disease. Aliment Pharmacol Ther. 2006;23:1455-1461.

36. Wyatt J, Vogelsang H, Hubl W, Waldhoer T, Lochs H. Intestinal permeability and the prediction of relapse in Crohn's disease. Lancet. 1993;341:1437-1439.

37. Shorter RG, Huizenga KA, Spencer RJ. A working hypothesis for the etiology and pathogenesis of nonspecific inflammatory bowel disease. Am J Dig Dis. 1972;17:1024-1032.

38. Renes IB, Verburg M, Van Nispen DJ, Buller HA, Dekker J, Einerhand AW. Distinct epithelial responses in experimental colitis: implications for ion uptake and mucosal protection. Am J Physiol Gastrointest Liver Physiol. 2002;283:G169-179.

39. Waldner MJ, Neurath MF. Master regulator of intestinal disease: IL-6 in chronic inflammation and cancer development. Semin Immunol. 2014;26:75-79.

40. Li L, Shi QG, Lin F, et al. Cytokine IL-6 is required in Citrobacter rodentium infection-induced intestinal Th17 responses and promotes IL-22 expression in inflammatory bowel disease. Mol Med Rep. 2014;9:831-836.

41. Galvez J. Role of Th17 cells in the pathogenesis of human IBD. ISRN Inflamm. 2014;2014:928461.

42. Shah C, Hari-Dass R, Raynes JG. Serum amyloid A is an innate immune opsonin for Gram-negative bacteria. Blood. 2006;108: 1751-1757.

43. Eckhardt ER, Witta J, Zhong J, et al. Intestinal epithelial serum amyloid A modulates bacterial growth in vitro and pro-inflammatory responses in mouse experimental colitis. BMC Gastroenterol. 2010;10:133.

44. Conti P, Bartle L, Barbacane RC, Reale M, Placido FC, Sipe J. Synergistic activation of serum amyloid A (SAA) by IL-6 and IL- 
1 in combination on human Hep 3B hepatoma cell line. Role of PGE2 and IL-1 receptor antagonist. Immunol Invest. 1995; 24:523-535.

45. Hagihara K, Nishikawa $\mathrm{T}$, Isobe $\mathrm{T}$, Song J, Sugamata $\mathrm{Y}$, Yoshizaki K. IL-6 plays a critical role in the synergistic induction of human serum amyloid A (SAA) gene when stimulated with proinflammatory cytokines as analyzed with an SAA isoform real-time quantitative RT-PCR assay system. Biochem Biophys Res Commun. 2004;314:363-369.

46. de Villiers WJ, Varilek GW, de Beer FC, Guo JT, Kindy MS. Increased serum amyloid a levels reflect colitis severity and precede amyloid formation in IL-2 knockout mice. Cytokine. 2000;12:1337-1347.

47. Zhong J, Eckhardt ER, Oz HS, Bruemmer D, de Villiers WJ. Osteopontin deficiency protects mice from dextran sodium sulfate-induced colitis. Inflamm Bowel Dis. 2006;12:790-796.

48. Cong Y, Brandwein SL, McCabe RP, et al. CD4 + T cells reactive to enteric bacterial antigens in spontaneously colitic $\mathrm{C} 3 \mathrm{H} /$ HeJBir mice: increased $\mathrm{T}$ helper cell type 1 response and ability to transfer disease. J Exp Med. 1998;187:855-864.

49. Contractor NV, Bassiri H, Reya T, et al. Lymphoid hyperplasia, autoimmunity, and compromised intestinal intraepithelial lymphocyte development in colitis-free gnotobiotic IL-2-deficient mice. J Immunol. 1998;160:385-394.

50. Dianda L, Hanby AM, Wright NA, Sebesteny A, Hayday AC, Owen MJ. T cell receptor-alpha beta-deficient mice fail to develop colitis in the absence of a microbial environment. Am J Pathol. 1997;150:91-97.
51. Garcia-Lafuente A, Antolin M, Guarner F, et al. Incrimination of anaerobic bacteria in the induction of experimental colitis. Am J Physiol. 1997;272:G10-15.

52. Kuhn R, Lohler J, Rennick D, Rajewsky K, Muller W. Interleukin-10-deficient mice develop chronic enterocolitis. Cell. 1993;75:263-274.

53. Rath HC, Schultz M, Freitag R, et al. Different subsets of enteric bacteria induce and perpetuate experimental colitis in rats and mice. Infect Immun. 2001;69:2277-2285.

54. Hudcovic T, Stepankova R, Cebra J, Tlaskalova-Hogenova H. The role of microflora in the development of intestinal inflammation: acute and chronic colitis induced by dextran sulfate in germ-free and conventionally reared immunocompetent and immunodeficient mice. Folia Microbiol. 2001;46:565-572.

55. Hans W, Scholmerich J, Gross V, Falk W. The role of the resident intestinal flora in acute and chronic dextran sulfate sodiuminduced colitis in mice. Eur J Gastroenterol Hepatol. 2000;12: 267-273.

56. Bodammer P, Zirzow E, Klammt S, Maletzki C, Kerkhoff C. Alteration of DSS-mediated immune cell redistribution in murine colitis by oral colostral immunoglobulin. BMC Immunol. 2013;14:10.

57. Stelwagen K, Carpenter E, Haigh B, Hodgkinson A, Wheeler TT. Immune components of bovine colostrum and milk. J Anim Sci. 2009;87:3-9.

58. Kehoe SI, Jayarao BM, Heinrichs AJ. A survey of bovine colostrum composition and colostrum management practices on Pennsylvania dairy farms. J Dairy Sci. 2007;90:4108-4116. 